\title{
Three-to-5-day weaning-to-estrus intervals do not affect neither efficiency of collection nor in vitro developmental ability of in vivo-derived pig zygotes
}

C. A. Martinez, J. M. Cambra, I. Parrilla, X. Lucas, Heriberto Rodriguez-Martinez, E. A. Martinez, J. C. Izpisua, C. Cuello and M. A. Gil

The self-archived postprint version of this journal article is available at Linköping University Institutional Repository (DiVA):

http://urn.kb.se/resolve?urn=urn:nbn:se:liu:diva-162303

N.B.: When citing this work, cite the original publication.

Martinez, C. A., Cambra, J. M., Parrilla, I., Lucas, X., Rodriguez-Martinez, H., Martinez, E. A., Izpisua, J. C., Cuello, C., Gil, M. A., (2020), Three-to-5-day weaning-to-estrus intervals do not affect neither efficiency of collection nor in vitro developmental ability of in vivo-derived pig zygotes,

Theriogenology, 141, 48-53. https://doi.org/10.1016/j.theriogenology.2019.09.004

Original publication available at:

https://doi.org/10.1016/j.theriogenology.2019.09.004

Copyright: Elsevier (12 months)

http://www.elsevier.com/

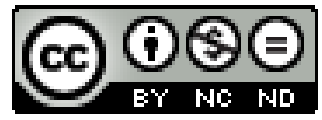


Three-to-5-day weaning-to-estrus intervals do not affect neither efficiency of collection nor in vitro developmental ability of in vivo-derived pig zygotes

CA Martinez ${ }^{\mathrm{a}, \mathrm{b}}$, JM Cambra ${ }^{\mathrm{a}, \mathrm{b}}$, I Parrilla ${ }^{\mathrm{a}, \mathrm{b}}, \mathrm{X} \mathrm{Lucas}^{\mathrm{a}, \mathrm{b}}, \mathrm{H}$ Rodriguez-Martinez ${ }^{\mathrm{c}}$, EA Martinez $^{\mathrm{a}, \mathrm{b}}$, JC Izpisua ${ }^{\mathrm{d}}, \mathrm{C}$ Cuello $^{\mathrm{a}, \mathrm{b}^{*}}, \mathrm{MA} \mathrm{Gil}^{\mathrm{a}, \mathrm{b}}$

aDepartment of Medicine and Animal Surgery, Faculty of Veterinary Medicine, International Excellence Campus for Higher Education and Research "Campus Mare Nostrum", University of Murcia, 30100, Murcia, Spain

${ }^{b}$ Institute for Biomedical Research of Murcia (IMIB-Arrixaca), Campus de Ciencias de la Salud, Carretera Buenavista s/n, 30120 El Palmar, Murcia, Spain

${ }^{c}$ Department of Clinical \& Experimental Medicine (IKE), Linköping University, Campus US, 58183, Linköping, Sweden

${ }^{\mathrm{d} S a l k}$ Institute for Biological Studies, 10010 N. Torrey Pines Rd., La Jolla, CA 92037 USA

*Corresponding author.

Cristina Cuello

Facultad de Veterinaria. Campus de Espinardo, 30100, Murcia, Spain

E-mail: ccuello@um.es

Tel.: +34868884734

Fax: +34868887069 


\section{Abstract}

An efficient system to collect large numbers of vital zygotes is a pre-requisite for application of zygote genome-editing technology, including development of efficient models for xenotransplantation using pigs. Owing to the sub-optimal in vitro production of zygotes in pigs, efficient collection of in vivo developed zygotes is required. Timing of ovulation is a key factor to sustain efficiency since the interval between pronuclear formation and the first division is very short in pigs. The weaning-to-estrus interval can, due to its inverse relation with length of estrus and time of ovulation, interfere with ovulation and make it asynchronous, which reduces the probability of obtaining zygotes. This retrospective study compared the effects of three weaning-to-estrus intervals of 3, 4 or 5 days on zygote collection efficiency in a total of 17 trials over a 3year period including 223 sows. Donor sows in groups of 10-15 animals were superovulated with eCG $24 \mathrm{~h}$ after weaning and those in estrus at $48-72 \mathrm{~h}$ post-eCG were immediately treated with hCG, followed by insemination 6 and $24 \mathrm{~h}$ thereafter. Collected structures during laparotomy on Day 2 (Day 0: onset of estrus) were morphologically evaluated and only those with a single cell and two visible polar bodies were considered as zygotes. Zygotes were injected with CRISPR-Cas9 editor mixture and cultured for 6 days to evaluate their developmental ability against non-injected control zygotes. Of all recovered structures ( $\mathrm{N}=5,468), 67.4 \%, 30.8 \%$ and $1.8 \%$ were zygotes, 2-cell embryos and oocytes-degenerated embryos, respectively. The different weaning-to-estrus intervals did not affect either the percentages of collected zygotes (range: $64.1 \%$ to $70.0 \%$ ) or the percentages of sows with zygotes at collection time (range: $69.0 \%$ to $73.3 \%$ ). The weaning-to-estrus intervals did not affect the in vitro developmental ability of zygotes. After $24 \mathrm{~h}$ of culture, $78.1 \pm 2.0 \%$ and $95.1 \pm 0.6$ $(\mathrm{P}<0.05)$ of injected $(\mathrm{N}=2,345)$ and non-injected $(\mathrm{N}=335)$ zygotes, respectively, developed to 2-to-4-cell embryo stage. The total efficiency of the system was $64.1 \pm 2.2 \%$ and $85.8 \pm 1.5 \%(\mathrm{P}<0.05)$ for injected and non-injected zygotes, respectively. In conclusion, the results indicate that neither the efficiency of collecting in vivo derived porcine zygotes from superovulated sows nor the zygote ability to develop to blastocyst after cytoplasmic genome-editing injection were affected by a weaning-to-estrus interval between 3-to-5 days.

Keywords: zygote, pig, embryo collection, CRISPR-Cas9, cytoplasmic injection, weaned to estrus interval 


\section{Introduction}

Zygote genome-editing engineering with programmable nucleases has initiated a revolution in agriculture and biomedical research, where pig is one of the most popular large animal models due to its similitude to humans in anatomy, organ size, physiology, and genomic and cell cycle characteristics [1]. Zygote genome-editing technology requires efficient methods to obtain large numbers of good-quality zygotes. Porcine zygotes can be produced by in vitro fertilization (IVF) of oocytes matured in vitro. However, and despite progress achieved in the last decades, current porcine IVF procedures remain inefficient and are associated with high levels of polyspermy and low embryo quality and blastocyst formation rates compared with in vivo-derived embryos [2-4]. For these reasons, in vivo-derivation is currently the main source of porcine zygotes for zygote genome-editing [5]. Surprisingly, information available on the efficiency of the procedures for collection of in vivo-derived porcine zygotes is scarce.

Post-weaned primiparous and multiparous sows have great potential as a zygote source for zygote genome-editing porcine programs, since weaning is an efficient physiological method of synchronizing estrus and ovulation in a group of sows. Most weaned sows exhibit a fertile estrus between 3 and 5 days post-weaning, with more than $90 \%$ exhibiting estrus within 7 days post-weaning [6,7]. In addition, superovulation with equine chorionic gonadotropin (eCG) $24 \mathrm{~h}$ after weaning followed by human chorionic gonadotropin (hCG) at the onset of estrus increases the number of viable embryos without affecting the number of unfertilized oocytes or the quality of the collected embryos [8].

Timing of ovulation is a key factor for the efficient recovery of in vivo zygotes. Since male and female pronuclei are formed 3 to $5 \mathrm{~h}$ post-fertilization, and the first division occurs 14-16 h later [9], zygote collection has to be accomplished over a very narrow window of time. In addition, there is a high variability in the ovulation time between individual sows [10], even after hCG treatment [11], which reduces the chance of obtaining embryos at the zygote stage during collection. In this context, the weaningto-estrus interval plays an important role because it has a strong inverse relationship with length of estrus and time of ovulation [6,10,12]. A weaning-to-estrus interval longer than 6 days or shorter than 2 days decreases reproductive performance of sows $[13,14]$ probably as consequence of alterations in the duration of estrus and time of ovulation. Moreover, high variability $(10 \mathrm{~h}$ to $60 \mathrm{~h})$ in the interval between the onset of 
estrus and period of ovulations has been also reported in sows with a weaning-to-estrus interval of 3 to 6 days [15]. For practical reasons, zygote collection programs normally use donor sows with a specific weaning-to-estrus interval (3 to 5 days) and these sows are usually grouped and not examined independently, which strongly limits our information on relevant embryo parameters.

Because these weaning-to-estrus intervals could still influence the duration of estrus and the timing of ovulations, this study compared the effects of three weaning-toestrus intervals (3, 4 or 5 days) on the efficiency of zygote collection in donor sows superovulated after weaning and on the in vitro developmental ability of genomeediting injected zygotes to achieve the blastocyst stage.

\section{Materials and Methods}

\subsection{Animals}

This work was conducted in a pig farm located in Southeastern Spain (Agropor S.L., Region of Murcia, Spain). Weaned sows (Landrace $\times$ Large-White) from 2 to 7 parities were selected as donors of zygotes. The sows were housed in individual gestation stalls in a mechanically ventilated facility. Mature boars (1-3 years of age) from a commercial artificial insemination station (AIM Iberica, Murcia, Spain) were used as semen donors. The animals were fed commercial diets according to their nutritional necessities and had ad libitum access to water. All the experimental procedures used in this study were performed in accordance with Directive 2010/63/EU EEC for animal experiments and were reviewed and approved by the Ethical Committee for Experimentation with Animals (code: 69/2014), the Research Ethics Committee (code: 1086/2015) of the University of Murcia, Spain; the Murcia Regional Ministry of Agriculture and Water (code: 273.705), and the Murcia Regional Ministry of Health (code: 061015), Spain.

\subsection{Superovulation, detection of estrus and insemination of donors}

Weaning was used to synchronize the estrus in donor sows. Only sows with a weaningto-estrus interval of 3 to 5 days were selected as donors. Superovulation was performed using eCG (1000 IU, im) (Foligon, Intervet, Boxmeer, The Netherlands) $24 \mathrm{~h}$ postweaning. Estrus was checked twice per day (07:00 h and 16:00 h) by snout-to-snout contact with a mature boar while applying manual backpressure. Sows with welldefined signs of estrus at $48-72 \mathrm{~h}$ post-eCG were treated with hCG (750 IU, im) 
(Veterin Corion, Divasa, Farmavic S.A., Barcelona, Spain) at the onset of estrus. Donors were post-cervically inseminated at 6 and $24 \mathrm{~h}$ after the onset of estrus with 1.5 $\times 10^{9}$ fresh spermatozoa extended in $45 \mathrm{~mL}$ of Beltsville thawing solution extender [16].

\subsection{Embryo collection and evaluation}

Zygote collections were carried out in an operating room inside the farm at 48-52 $\mathrm{h}$ after onset of estrus. Sows were sedated with azaperone $(2 \mathrm{mg} / \mathrm{kg}$ body weight, im) and anesthetized with sodium thiopental ( $7 \mathrm{mg} / \mathrm{kg}$ body weight, iv). Anesthesia was maintained with isofluorane (3-5\% in air) and buprenorphine $(0.3 \mathrm{mg} / \mathrm{sow}$, im) was used as post-surgical analgesic drug. The genital tract was exposed through mid-ventral laparotomy, and the number of corpora lutea on each ovary was counted. Zygotes were retrieved by flushing each oviduct with $30 \mathrm{~mL}$ of modified Tyrode's lactate-HEPESpolyvinyl alcohol medium (TL-HEPES-PVA) [11,17]. Collected structures were washed, placed in tubes containing $1.5 \mathrm{~mL}$ of the same medium and transported at $39{ }^{\circ} \mathrm{C}$ to our laboratory at the University of Murcia within $1 \mathrm{~h}$ after collection. Embryos were then carefully assessed under a stereomicroscope at a magnification of $60 \mathrm{x}$ and only embryos with a single cell and two visible polar bodies were considered as zygotes.

\subsection{Intracytoplasmic injection of zygotes}

Zygotes were injected by using the laser-assisted cytoplasmic microinjection protocol previously described by [18]. Briefly, the zygote was immobilized by suction with a holding pipette and positioned with the polar bodies at either 6 or 12 o'clock. Then a hole was created in the zona pellucida at 3 o'clock position by using a laser and an injection pipette was inserted through the hole and advanced into the zygote until the plasma membrane was broken by aspiration of ooplasm into the injection pipette. Then, the aspirated ooplasm and the editor mixture $(100 \mathrm{ng} / \mu \mathrm{L}$ Cas9 mRNA and $20 \mathrm{ng} / \mu \mathrm{L}$ sgRNA) were injected into the cytoplasm.

\subsection{In vitro embryo culture and assessment of in vitro embryo development}

After injections, zygotes were placed into a 4-well multi-dish (40 zygotes per well) containing $500 \mu \mathrm{L}$ of NCSU-23 medium [19] supplemented with $0.3 \mathrm{mM}$ pyruvate and $4.5 \mathrm{mM}$ lactate for $24 \mathrm{~h}$ and then transferred to fresh NCSU-23 medium supplemented with $5.5 \mathrm{mM}$ glucose for 5 additional days. Embryos were cultured at $39{ }^{\circ} \mathrm{C}, 5 \% \mathrm{CO}_{2}$ in 
air and 95-97\% relative humidity. At Day 5, culture medium was supplemented with $10 \%$ fetal calf serum. Embryo development was assessed under a stereomicroscope at $24 \mathrm{~h}$ and $144 \mathrm{~h}$ of culture to determine cleavage and blastocyst formation rates, respectively.

\subsection{Experimental design}

In this retrospective study, data related to the zygote collection was recorded over a 3year period in 17 trials using a total of 223 sows with a lactation length of $21.8 \pm 0.1$ days and good body condition and reproductive history. In each trial, groups of 10-15 sows with a weaning-to-estrus interval of 3, 4 or 5 days were selected as donors. Donors from the three groups in each trial were inseminated with sperm doses from the same boar. To evaluate the effect of the weaning-to-estrus intervals on the efficiency of zygote collection, the numbers of viable zygotes, 2-cell embryos and oocytes/degenerated embryos recovered in each donor were counted. Recovery rate was defined as the ratio of the number of embryos and oocytes/degenerated embryos recovered to the number of corpora lutea present. Fertilization rate was defined as the ratio of the number of viable embryos to the total number of embryos and oocytes/degenerated embryos collected. Within each group, zygotes collected from each sow were genome-editing injected and cultured in vitro to evaluate their ability for blastocyst development compared to control non-injected zygotes. An embryo that had cleaved to the 2-to-4-cell stage was defined as cleaved, and an embryo with a welldefined blastocoel and an inner cell mass and trophoblast totally discernible was defined as a blastocyst. The cleavage rate was the percentage of zygotes that had divided to the 2-to-4-cell stage. Blastocyst formation was the percentage of 2-to-4-cell embryos that developed to the blastocyst stage. The total efficiency was described as the percentage of the total number of cultured zygotes that reached the blastocyst stage.

\subsection{Statistical analysis}

The data were analysed with the IBM SPSS 24.0 Statistics package (IBM, Chicago, IL, USA). Percentage data were compared using Fisher's exact test. The continuous variables were evaluated using the Kolmogorov-Smirnov test for normality and groups were compared by ANOVA or Student's t-test, as appropriate. The ANOVA model included the main effect of weaning-to-estrus interval and the random effect of the replicate. When the ANOVA indicated a significant effect, the means were compared 
by Bonferroni's test. Differences were considered significant at $\mathrm{P}<0.05$. The results are presented as mean $\pm \mathrm{SEM}$.

\section{Results}

A total of 217 out of 223 donors (97.3\%) had embryos at collection. Neither mean ovulation rate $(27.3 \pm 0.5$ corpora lutea) nor the mean number of structures $(25.2 \pm 0.6)$ collected in these sows differed between weaning-to-estrus interval groups $(92.3 \pm 1.0 \%$ total recovery rate) (Fig. 1). Of all recovered structures $(\mathrm{N}=5,468), 67.4 \pm 2.9 \%$, $30.8 \pm 2.8 \%$ and $1.8 \pm 0.4 \%$ were zygotes, 2-cell embryos and oocytes-degenerated embryos, respectively $(98.2 \%$ fertilization rate). The different weaning-to-estrus intervals did neither affect the percentages of collected zygotes (range: $64.1 \%$ to $70.0 \%$ ) (Fig. 2) nor the percentages of sows with zygotes at the collection (range: $69.0 \%$ to 73.3\%) (Fig. 3). Table 1 shows the total efficiency of the procedure.

The developmental competence of injected zygotes to the blastocyst stage is shown in Table 2. The weaning-to-estrus intervals did not affect the in vitro developmental ability of injected zygotes. After $24 \mathrm{~h}$ of culture, $78.1 \pm 2.0 \%$ and $95.1 \pm 0.6(\mathrm{P}<0.05)$ of injected and non-injected zygotes, respectively, developed to 2-to4-cell embryo stage and $82.0 \pm 1.6 \%$ and $90.2 \pm 0.7 \%$ of them, respectively, achieved the blastocyst stage by day 6 of culture. The total efficiency of the system was $64.1 \pm 2.2 \%$ for injected and $85.8 \pm 1.5 \%$ for non-injected control zygotes $(\mathrm{P}<0.05)$ (Fig. 4).

\section{Discussion}

The present study demonstrates that weaning-to-estrus intervals between 3 and 5 days do not affect neither the efficiency of collecting in vivo derived porcine zygotes nor the zygote ability to develop to blastocyst after cytoplasmic injection and further in vitro culture for 6 days. In addition, herein we describe an efficient protocol for obtaining high-quality porcine zygotes from post-weaning multiparous sows.

To develop a practical and effective protocol for zygote collection, we decided to use in vivo derived zygotes and post-weaning multiparous sows as embryo donors by two main reasons: firstly, although the production of porcine zygotes in vitro may be a good alternative with lower costs and shorter time compared with in vivo collection, the current IVF systems remain sub-optimal; and secondly, although zygotes can be obtained in vivo from prepubertal and pubertal gilts, we chose multiparous sows firstly because superovulation treatments in prepubertal gilts and synchronized mature gilts 
produced high proportions (25-50\%) of poor quality embryos, unfertilized oocytes and degenerated embryos [20-23] as well as increased embryonic losses post-implantation $[24,25]$. It was suggested that a percentage of ovulated oocytes in synchronizedsuperovulated females remains in the germinal vesicle stage (immature) and would not be fertilized [26,27]. To confirm this speculation, we collected over $40 \%$ of immature oocytes on day 6 of the cycle from pubertal gilts superovulated with $1000 \mathrm{IU}$ eCG at the end of the synchronization treatment, in previous experiments (unpublished), Together, these factors make inadvisable the use of these female categories as embryo donors. Secondly, superovulation with eCG $24 \mathrm{~h}$ after weaning followed by a dose of hCG at the onset of estrus markedly increased the number of viable embryos without negative effects on the number of unfertilized oocytes or the quality of the collected embryos [8]. Moreover, high reproductive performance of recipients was observed after non-surgical transfers of superovulated embryos collected from post-weaning sows $[8,28]$, indicating that these embryos retained their potential to develop to term.

In the present study, we only used weaned 2 to 7 parity sows as donors due to their high accessibility on-farm and because they usually have farrowing rates and litter sizes higher than those of primiparous sows. Moreover, primiparous sows have longer weaning-to-estrus intervals than multiparous sows [29-31], which is associated with differences in duration of estrus and timing of ovulation [13]. Differences in these parameters between primiparous and multiparous sows could be large enough to have important implications for the programming of protocols for zygote collection.

We only selected donor sows with weaning-to-estrus intervals between 3-to-5 days because more than $80 \%$ of the superovulated donor sows start estrus within that period after weaning [8]. Therefore, the laparotomies for collecting embryos (which were performed in all cases at day 2 after onset of estrus) could be concentrated in only three consecutive days in each trial. Although weaning-to-estrus interval play an important role in the duration of estrus and hence in the time of ovulations [10], the results of the present study indicate that weaning-to-estrus intervals between 3-to-5 days did not affect the percentage of sows with zygotes at surgical collection, which suggest that such weaning-to-estrus intervals did not influence the period of ovulations. This apparent discrepancy can be justified by the extension of the weaning-to-estrus interval. Thus, while short ( $<2$ days) or long ( $>6$ days) weaning-to-estrus intervals decrease reproductive performance of sows $[13,14]$ by increasing the probability of too early or 
post-ovulatory inseminations, weaning-to-estrus intervals between 3 and 5 days were associated to high fertility and prolificacy [32].

Embryos at the zygote stage could be collected from approximately $60 \%$ of the sows and $30 \%$ and $10 \%$ of the remaining sows had cleaved embryos and a mixed of zygotes and cleaved embryos at collection, respectively. These results indicate that despite of the weaning and superovulation treatment used, ovulations occurred before than expected in some sows. Although variability in the ovulation period among sows is reported [10], even among sows with weaning-to-estrus intervals from 3 to 5 days [15], other factors could be implicated. In our study, the estrus detection was performed twice per day and that it is likely that some sows diagnosed in estrus in the morning could really had started estrus even $12 \mathrm{~h}$ earlier, which would affect ovulation time and, consequently, the developmental stage of the embryos at collection.

Our results also indicate that weaning-to-estrus intervals did not affect the developmental ability of the zygotes to achieve the blastocyst stage. In addition, with the protocol described here, we obtained a high global efficiency of blastocyst formation (number of blastocysts formed to the number of zygotes used) ( $\sim 64 \%)$. That efficiency was much higher than those previously reported using parthenogenetic embryos [33,34] or IVF presumed zygotes [35] where the efficiency of blastocyst formation rates ranged between $10 \%$ and $15 \%$. These results indicate that the number of blastocysts formed from in vivo derived zygotes may be approximately six times greater compared to those formed from in vitro produced embryos.

In the present study, the developmental ability of injected zygotes $(\sim 64 \%)$ was lower than that of non-injected counterparts ( $\sim 85 \%)$, suggesting that zygote cytoplasmic injection had an adverse effect on further embryonic development. This can be due to differences in the sensitivity to the culture conditions between injected and non-injected zygotes, as well as the culture environment more easily affects nuclear transfer than IVF produced embryos [36]. Our finding disagree with those obtained using parthenogenetic and IVF embryos where no differences in embryo development were reported between genome-editing injected and control non-injected 1-cell embryos [33-35]. Differences in the sensitivity to manipulation during genome-editing injection or to culture conditions between in vitro produced and in vivo developed zygotes could be responsible for that discrepancy.

\subsection{Conclusions}


In conclusion, this study clearly demonstrates that weaning-to-estrus interval (3 to 5 days) did not affect neither the percentages of sows with embryos at the zygote stage at the collection nor the number and quality of the collected zygotes. After genome-editing injection, $80 \%$ of the zygotes cleaved by $24 \mathrm{~h}$ of culture and $80 \%$ of them developed to the blastocyst stage by day 6 of culture. The protocol used in this study was very reliable through the different trials $(n=17)$ and could be used in multiple applications where high-quality zygotes are needed. Post-weaning sows could be super-ovulated and inseminated on (commercial) farms and the laparotomies for zygote collection could be performed on the same farm or after transportation of the animals to an experimental unit. Because the donors can be healthy weaned sows with five or more parities, just before they are culled for farm strategies, the cost of the animals is low and the available number of animals is high.

\section{Acknowledgments}

The authors are grateful to Alicia Nohalez and Moises Gonzalvez for their assistance throughout this work. We also thank the staff of Agropor SA (Murcia, Spain) and Porcisan (Murcia, Spain) piggeries for the excellent management of the animals. CAM is supported by a postdoctoral grant from the Fundación Séneca, (Murcia, Spain; 20780/PD/18) and JMC is supported by a predoctoral grant from the Ministry of Economy and Competitiveness (Madrid, Spain; BES-2016-077869). Work in the laboratory of JCI was supported by the UCAM, G. Harold and Leila Y. Mathers Charitable Foundation, The Moxie Foundation and The Leona M. and Harry B. Helmsley Charitable Trust (2012-PG-MED002).

\section{Funding}

This study was supported by the Seneca Foundation, Murcia, Spain (grant number 19892/GERM/15) and the UCAM (Murcia, Spain).

\section{Role of the funding source}

Funding sources did not have any involvement in the study design, in the collection, analysis and interpretation of data, in the writing of the report, and in the decision to submit the article for publication.

\section{Author contributions}


JC Izpisua and EA Martinez conceived and designed the study. EA Martinez, H Rodriguez-Martinez, C Cuello and MA Gil directed the experiments. CA Martinez, JM Cambra, I Parrilla, X Lucas, EA Martinez, C Cuello and MA Gil performed the experiments. CA Martinez, JM Cambra, I Parrilla, EA Martinez, H Rodriguez-Martinez,

$\mathrm{C}$ Cuello and MA Gil analysed and interpreted the data. CA Martinez wrote the manuscript. I Parrilla, EA Martinez, H Rodriguez-Martinez, JC Izpisua, C Cuello and MA Gil revised and discussed the manuscript. All authors read and approved the manuscript for publication. JC Izpisua, MA Gil and EA Martinez secured funding.

\section{Declaration of interest}

None of the authors have any conflicts of interest to declare.

\section{References}

[1] Perleberg C, Kind A, Schnieke A. Genetically engineered pigs as models for human disease. Dis Model Mech 2018; 11(1)

[2] Gil MA, Cuello C, Parrilla I, Vazquez JM, Roca J, Martinez EA. Advances in swine in vitro embryo production technologies. Reprod Domestic Anim 2010; 45:40-8.

[3] Grupen CG. The evolution of porcine embryo in vitro production. Theriogenology 2014; 81:24-37.

[4] Martinez CA, Cambra JM, Maside C, Cuello C, Roca J, Martinez EA, et al. High pre-freezing sperm dilution improves monospermy without affecting the penetration rate in porcine IVF. Theriogenology 2019; 131:162-8.

[5] Gil MA, Martinez CA, Nohalez A, Parrilla I, Roca J, Wu J, et al. Developmental competence of porcine genome-edited zygotes. Mol Reprod Dev 2017; 84:814-21.

[6] Belstra BA, Flowers WL, See MT. Factors affecting temporal relationships between estrus and ovulation in commercial sow farms. Anim Reprod Sci 2004; 84:377-94.

[7] Behan JR, Watson PF. The effect of managed boar contact in the postweaning period on the subsequent fertility and fecundity of sows. Anim Reprod Sci $2005 ; 88: 319-24$.

[8] Angel MA, Gil MA, Cuello C, Sanzhez-Osorio J, Gomis J, Parrilla I, et al. The effects of superovulation of donor sows on ovarian response and embryo 
development after nonsurgical deep-uterine embryo transfer. Theriogenology $2014 ; 81: 832-9$.

[9] Hunter RHF. Chronological and cytological details of fertilization and early embryonic development in the domestic pig sus escrofa. Anat Rec 1974; 178:169-86.

[10] Kemp B, Soede NM. Relationship of weaning-to-estrus interval to timing of ovulation and fertilization in sows. J Anim Sci 1996; 74:944-9.

[11] Martinez EA., Angel MA, Cuello C, Sanchez-Osorio J, Gomis J, Parrilla I, et al. Successful non-surgical deep uterine transfer of porcine morulae after 24 hour culture in a chemically defined medium. PLoS ONE 2014; 9(8).

[12] Almeida FR, Novak S, Foxcroft GR. The time of ovulation in relation to estrus duration in gilts. Theriogenology 2000; 53:1389-96.

[13] Soede NM, Kemp B. Expression of oestrus and timing of ovulation in pigs. J Reprod Fertil Suppl. 1997; 52:91-103.

[14] Poleze E, Bernardi ML, Amaral Filha WS, Wentz I, Bortolozzo FP. Consequences of variation in weaning-to-estrus interval on reproductive performance of swine females. Livest Sci 2006; 103:124-30.

[15] Soede NM, Wetzels CC, Zondag W, de Koning MA, Kemp B. Effects of time of insemination relative to ovulation, as determined by ultrasonography, on fertilization rate and accessory sperm count in sows. J Reprod Fertil 1995; 104:99-106.

[16] Pursel VG, Johnson LA. Freezing of boar spermatozoa: fertilizing capacity with concentrated semen and a new thawing procedure. J Anim Sci 1975; 40: 99-102.

[17] Funahashi H, Ekwall H, Rodriguez-Martinez H. Zona reaction in porcine oocytes fertilized in vivo and in vitro as seen with scanning electron microscopy. Biol Reprod 2000; 63:1437-42.

[18] Bogliotti YS, Vilarino M, Ross PJ. Laser-assisted cytoplasmic microinjection in livestock zygotes. J Vis Exp 2016; 5:116.

[19] Peters RM, Wells KD. Culture of pig embryos. J Reprod Fertil 1993; 48:6173.

[20] Guthrie HD, Henricks DM, Handlin DL. Plasma hormone levels and fertility in pigs induced to superovulate with PMSG. J Reprod Fertil 1974; 41:361-70. 
[21] Holtz W and Schlieper B 1991. Unsatisfactory results with the transfer of embryos from gilts superovulated with PMSG and hCG. Theriogenology 35, 1237-1249.

[22] Wallenhorst S, Holtz W. Embryo collection in prepubertal gilts and attempts to develop an improved embryo transfer technique. Vet Rec 2002; 150:749-51.

[23] Ziecik AJ, Biallowicz M, Kaczmarek M, Demianowicz W, Rioperez J, Wasielak et al. Influence of estrus synchronization of prepubertal gilts on embryo quality. J Reprod Dev 2005; 51:379-84.

[24] Martinat-Botté F, Venturi E, Guillouet P, Driancourt MA, Terqui M. Induction and synchronization of ovulations of nulliparous and multiparous sows with an injection of gonadotropin-releasing hormone agonist (Receptal). Theriogenology 2010; 73:332-42.

[25] Furstoss V, Berthelot F, Plat M, Venturi E, Royer E, Elleboudt F et al. Genetic and non-genetic parameters related to embryo production in superovulated Large White (LW) gilts. Anim Reprod Sci 2012; 134:177-83.

[26] Estienne MJ, Harper AF, Horsley BR, Estienne CE, Knight JW. Effects of P.G. 600 on the onset of estrus and ovulation rate in gilts treated with Regumate. J Anim Sci 2001; 79:2757-61.

[27] Horsley BR, Estienne MJ, Harper AF, Purcell SH, Baitis HK, Beal WE, et al. Effect of P.G. 600 on the timing of ovulation in gilts treated with altrenogest. J Anim Sci 2005; 83:1690-5.

[28] Hazeleger W, Bouwman EG, Noordhuizen JPTM, Kemp B. Effect of superovulation induction on embryonic development on day 5 and subsequent development and survival after nonsurgical embryo transfer in pigs. Theriogenology 2000; 53:1063-70.

[29] Hurtgen JP, AD Leman, Crabo B. Seasonal influence on estrous activity in sows and gilts. J Am Vet Med A 1980; 176:119-23.

[30] Clark LR, Komkov A, Tribble LF. Effects of parity, season, gonadotropin releasing hormone and altered suckling intensity on the interval to rebreeding in sows. Theriogenology 1986; 26:299-308.

[31] Koketsu Y, Dial GD. Factors influencing the postweaning reproductive performance of sows on commercial farms. Therionology 1997; 47:1445-61.

[32] Martinez EA, Vazquez JM, Parrilla I, Cuello C, Gil MA, Rodriguez-Martinez $\mathrm{H}$, et al. Incidence of unilateral fertilizations after low dose deep intrauterine 
insemination in spontaneously ovulating sows under field conditions. Reprod Domest Anim 2006; 41:41-7.

[33] Wang Y, Du Y, Shen B, Zhou X, Li J, Liu Y, et al. Efficient generation of gene-modified pigs via injection of zygotes with Cas9/sgRNA. Sci Rep 2015; 5:8256.

[34] Zhou X, Wang L, Du Y, Sie F, Li L, Liu Y, et al. Efficient generation of genemodified pigs harboring precise orthologous human mutation via CRISPR/Cas9-induced homology-directed repair in zygotes. Hum Mutat 2016; $37: 110-8$.

[35] Whitworth KM, Rowland RRR, Ewen CL, Trible BR, Kerrigan MA, CinoOzuna AG, et al. Gene-edited pigs are protected from porcine reproductive and respiratory syndrome virus. Nat Biotechnol 2016; 34:20-2.

[36] Yamanaka K, Sugimura S, Wakai T, Kawahara M, Sato E. Difference in sensitivity to culture condition between in vitro fertilized and somatic cell nuclear transfer embryos in pigs. J Reprod Dev 2009; 55:299-304. 


\section{Figure legends}

Figure 1. Influence of weaning-to-estrus-interval (WEI) on the reproductive parameters in fertilized donor sows.

Figure 2. Influence of weaning-to-estrus-interval (WEI) on the frequency distribution of zygotes, 2-cell embryos and oocytes retrieved from donor sows.

Figure 3. Influence of weaning-to-estrus-interval (WEI) on the frequency distribution of sows with embryos at different stages at the collection.

Figure 4. In vitro developmental efficiency of in vivo derived zygotes microinjected with Cas9 mRNA/sgRNA 
Table 1. Global efficiency of the protocol used for zygote collection from superovulated weaned sows $(n=217)$

Parameter

Corpora lutea (mean \pm SEM) $27.3 \pm 0.5$

Oocytes and embryos collected (mean \pm SEM) $25.2 \pm 0.6$

Percentage of zygotes collected (\%) 67.4

Percentage of 2-cell embryos collected (\%) 30.8

Percentage of oocytes and degenerated collected (\%) 1.8

Sows with only zygotes (\%) 59.4

Sows with zygotes and 2-cell embryos (\%) 12.4

Sows with only 2-cell embryos (\%) 28.1 
Table 2. Influence of weaning to estrus interval on the in vitro developmental ability of in vivo derived zygotes microinjected with Cas 9 mRNA/sgRNA

\begin{tabular}{ccccc}
\hline $\begin{array}{c}\text { Weaned to } \\
\text { estrus interval } \\
(\text { days })\end{array}$ & $\begin{array}{c}\text { Injected } \\
\text { zygotes } \\
(\mathrm{N})\end{array}$ & $\begin{array}{c}\text { Cleavage rate } \\
\mathrm{N}(\text { mean } \pm \mathrm{SEM})^{\#}\end{array}$ & $\begin{array}{c}\text { Blastocyst formation rate } \\
\mathrm{N}(\text { mean } \pm \text { SEM })^{\#}\end{array}$ & $\begin{array}{c}\text { Efficiency rate }^{* *} \\
\mathrm{~N}(\text { mean } \pm \mathrm{SEM})^{\#}\end{array}$ \\
\hline 3 & 799 & $620(78.6 \pm 3.4)$ & $512(84.0 \pm 3.0)$ & $512(63.6 \pm 3.5)$ \\
4 & 1312 & $1009(77.5 \pm 2.9)$ & $840(82.4 \pm 1.9)$ & $840(64.4 \pm 3.1)$ \\
5 & 234 & $195(81.3 \pm 4.8)$ & $149(79.9 \pm 3.6)$ & $149(63.0 \pm 4.8)$ \\
\hline
\end{tabular}

${ }^{*}$ Number of blastocysts/total of cleaved embryos. ${ }^{* *}$ Number of blastocyst/total of zygotes injected. ${ }^{\#}$ Mean \pm SEM from 17 trials. 